\title{
Digitale Transformation im Vormarsch
}

Das Interesse der produzierenden Unternehmen an maschinellem Lernen (ML), Künstlicher Intelligenz (KI), dem Internet-of-Things (IoT), digitalen Zwillingen und robotergesteuerter Prozessautomatisierung nimmt kontinuierlich zu. Diese Technologien sind vielfach produktiver beim Lösen einzelner Aufgaben, entfalten ihr volles Potenzial aber erst, wenn sie nicht in geschlossenen Räumen erdacht und angewendet werden, sondern in Geschäftsprozessen vernetzt zur Wertschöpfung beitragen. Unternehmen erzielen den größten Nutzen durch die Kombination der unterschiedlichen Anwendungsmöglichkeiten und durch Kopplung von innovativen Automatisierungstechnologien mit neu gestalteten Betriebsabläufen. Der Digitalisierungsschub während der Pandemie hat dazu beigetragen, dass viele Unternehmen sich nun trauen, digitale Technologien intensiv einzusetzen und damit ihre Geschäftsprozesse zu transformieren.

Die Führungskräfte erkennen immer mehr, dass die digitale Transformation wesentlich zur Zukunftssicherheit ihrer Unternehmen beiträgt. Das gilt insbesondere dann, wenn nicht mehr ein „Entweder-Oder“, sondern ein Miteinander von Mensch und Maschine gelebt wird. Die leistungsstärksten Unternehmen haben inzwischen gelernt, wie sie digitale Technologien einbetten können, um die menschliche Produktivität zu steigern. So wird beispielsweise verstärkt in KIbasierte Konversationsapplikationen investiert. Diese sollen die Mitarbeitenden bei ihrer jeweiligen Aufgabe befähigen und unterstützen, um die Produktivität, Anpassungsfähigkeit und Entscheidungsfindung im Unternehmen zu erhöhen. Einer Prognose zufolge werden zukünftig sogar 50 Prozent der Wissenschaftler regelmäßig mit ihrem eigenen KI-gestützten Roboterassistenten interagieren, der ihnen dabei hilft, Aufgaben zu identifizieren und zu priorisieren, Informationen zu sammeln und sich wiederholende Arbeiten zu automatisieren.

Durch dieses Zusammenwachsen von Informationstechnologien (IT) und operativen Technologien (OT) wandeln sich Unternehmen in „digitale Innovationsfabriken“. Analysten von IDC erwarten, dass 40 Prozent der marktführenden IT- und OT-Anbieter strategische Partnerschaften eingehen werden, um eine ganzheitliche Lösung anzubieten. Aber vielleicht liegt genau darin ein Differenzierungspotenzial für die Unternehmen, um kommerzielle Lösungen nicht einfach standardmäßig anzuwenden, sondern als neue Kernkompetenz intern zu adaptieren und weiterzuentwickeln.

Ihre ZWF-Herausgeber und-Redaktion

Predictive Maintenance - Technologieansätze und Geschäftsmodelle

Technologische Trends wie Digitalisierung und Industrie 4.0 frühzeitig zu erkennen und zielführend nutzbar zu machen entwickelt sich zu einer wettbewerbskritischen Kompetenz. TIM Consulting sowie LAPP verstehen sich hier als Enabler, die ihren Kunden mit passgenauen und zukunftsorientierten Lösungen zur Seite stehen.

Am Beispiel Predictive Maintenance und der dafür entwickelten Praktiker-Guideline zeigen die beiden Unternehmen auf, wie technologische Potenziale exploriert und implementiert werden können. TIM
Consulting bringt dabei seine Kompetenzen in den Bereichen Technologieexploration und Foresighting ein, LAPP seine Expertise bei Verbindungstechnologien in der Industrieautomatisierung. Lesen Sie mehr dazu im Beitrag in diesem Heft (S. 667-671).

Kontakt:

Dr. Patrick Olivan (www.lapp.com)

Dr. Ulrich Hutschek

(www.tim-consulting.de)

Dr. Silvia Rummel

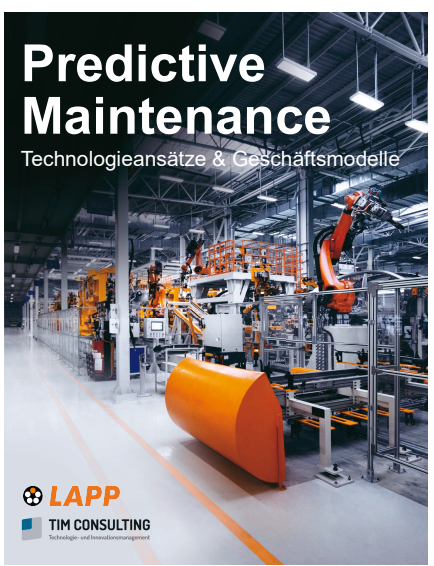

